\title{
3 Research Square \\ COVID-19 presented with Deep Vein Thrombosis: An unusual case report
}

\section{Lotfollah Davoodi}

Microbial Resistance Research Center, Mazandaran University of Medical Sciences, Sari

https://orcid.org/0000-0001-7298-7789

\section{Morteza Taghavi}

Cardiovascular Research Center, Mazandaran University of Medical Sciences, Sari

\section{Alireza Razavi ( $\nabla$ razavialireza33@gmail.com )}

Student Research Committee, Faculty of Medicine, Mazandaran University of Medical Sciences, Sari, Iran. https://orcid.org/0000-0002-4386-4672

\section{Case Report}

Keywords: COVID-19, DVT, pulmonary thromboembolism, bilateral patchy GGO

Posted Date: April 7th, 2020

DOI: https://doi.org/10.21203/rs.3.rs-21602/v1

License: (1) (1) This work is licensed under a Creative Commons Attribution 4.0 International License. Read Full License 


\section{Abstract}

\section{Background}

On 31 December 2019, the World Health Organization (WHO) was informed of a cluster of cases of pneumonia of unknown cause detected in Wuhan City, Hubei Province, China. The pneumonia was caused by a virus called SARS-Cov-2, which was later named COVID-19. In this report, we present a patient with COVID-19 who developed deep vein thrombosis.

\section{Case presentation}

A 57-year-old woman presented to the clinic's infectious department with no underlying illness due to pain, redness, and leg swelling. According to a patient report, she had a mild dry cough for the past 3 days and had no other symptoms. The patient had no history of prone thrombosis conditions. Initially, CT angiography was performed to rule out pulmonary thromboembolism, which showed no evidence of thrombosis. Dilatation and thrombosis were seen in the examinations of the paired veins of the leg, popliteal, superficial and left femoral joints, and no evidence of vascular flow suggesting acute DVT. Because of fever and lymphopenia, nasal swabs were used for sampling and SARS-CoV-2 nucleic acid was detected by RT-PCR. Chest X-ray also revealed bilateral patchy ground-glass opacity. Other tests including ANA, Anti-dsDNA, RF test and ACA test was normal. Heparin at a dose of 80 units $/ \mathrm{kg}$ IV bolus, chloroquine $400 \mathrm{mg}$ single dose and lopinavir/ritonavir (Kaletra) $400 \mathrm{mg}$ twice daily were prescribed to treat illness and relieve symptoms. On illness day 3, fever stopped and nasal swab sample turned undetectable for SARS-CoV-2 by RT-PCR as well as swelling and tenderness on her leg had been disappeared gradually. She is under regular follow-up with no new symptoms to date.

\section{Conclusion}

The mechanism of DVT formation due to COVID-19 is unknown despite thrombocytopenia, and has not been investigated but it resolved as COVID-19 symptoms, tenderness, and leg pain improved. Although COVID-19 presented with Deep Vein Thrombosis is a rare condition, in middle-aged people with sudden onset of manifestations, we should recognize it from other diseases as an important and treatable differential diagnosis. Rapid diagnostic assays, efficient treatment, and prudent use of CT-scan are important to control future COVID-19 spread.

\section{Background}

On 31 December 2019, the World Health Organization (WHO) was informed of a cluster of cases of pneumonia of unknown cause detected in Wuhan City, Hubei Province, China. The pneumonia was caused by a virus called SARS-Cov-2, which was later named COVID-19. Symptoms are similar to the common cold, most notably fever and dyspnea. $[1,2]$. The disease is highly contagious so that in the WHO's 51st situational report on March 11, 2020, announced a total of 118319 people and 4292 deaths [3]. In this report, we present a patient with COVID-19 who developed deep vein thrombosis. 


\section{Case Presentation}

A 57-year-old woman presented to the clinic's infectious department with no underlying illness due to pain, redness, and leg swelling. The patient had no history of prone thrombosis conditions. According to a patient report, she had a mild dry cough for the past 3 days and had no other symptoms. On examination, a mild fever of $38.1^{\circ}$ and at the touch of the leg, there was tenderness in the paired vein path of the leg and swelling of the leg with a difference of $6 \mathrm{~cm}$ on both sides. Initially, CT angiography was performed to rule out pulmonary thromboembolism, which showed no evidence of thrombosis. With the assumption of Deep Vein Thrombosis, left lower limb venous color Doppler ultrasound was performed. Dilatation and thrombosis were seen in the examinations of the paired veins of the leg, popliteal, superficial and left femoral joints, and no evidence of vascular flow suggesting acute DVT. Thrombosis spread to the external iliac and left iliac veins up to the level of the bifurcation of the common iliac veins, as well as thrombosis to the superficial and small saphenous veins. There was no evidence of thrombus infiltration into the inferior vena cava. In the patient's blood tests, revealed elevated LDH (655 U/L, normal: 140-280 $\mathrm{U} / \mathrm{L}$ ), elevated CRP level (47 mg/L, normal: $<10 \mathrm{mg} / \mathrm{L})$, elevated aspartate aminotransferase (AST, $59 \mathrm{U} / \mathrm{L}$, normal: $10-40 \mathrm{U} / \mathrm{L}$ ), and thorombocytopenia. White-cell count was 2300 per microliter (with $65.7 \%$ neutrophils and $23 \%$ lymphocytes). Because of fever and lymphopenia, nasal swabs were used for sampling and SARS-CoV-2 nucleic acid was detected by RT-PCR. Other tests including ANA, Anti-dsDNA, RF test, Anti-cardiolipin antibodies (ACA), Factor V Leiden, and S, C protein test was normal. Chest X-ray also revealed bilateral patchy ground-glass opacity. After the definitive diagnosis of COVID-19 which presented with DVT in the patient, she was admitted on $17^{\text {th }}$ March and medication and supportive treatment were initiated. SPO2 was $90 \%$ when the patient was breathing with a nasal catheter. Heparin at a dose of 80 units $/ \mathrm{kg}$ IV bolus, then continuous infusion of $18 \mathrm{units} / \mathrm{kg} / \mathrm{hr}$, chloroquine $400 \mathrm{mg}$ single dose and lopinavir/ritonavir (Kaletra) $400 \mathrm{mg}$ twice daily were prescribed to treat illness and relieve symptoms. On illness day 3, fever stopped and nasal swab sample turned undetectable for SARS-CoV-2 by RT-PCR. The results of subsequent RT-PCR of nasal swabs on illness days 7 and 10, respectively, were repeatedly negative as well as swelling and tenderness on her leg had been disappeared gradually. On day 7 , oxygen therapy was discontinued so that the patient's SPO2 was $93 \%-98 \%$ at room air pressure. After getting good results with no side effects, rivaroxaban (10 mg PO) was started and heparin was discontinued and finally, she was discharged. She is under regular follow-up with no new symptoms to date.

\section{Discussion And Conclusion}

The most important clinical symptoms in COVID-19 patients are fever, dry cough and shortness of breath. In our case, unlike other studies, Deep Vein Thrombosis was observed with no history of thrombosis [1]. One study has shown that thrombocytopenia is associated with COVID-19 infection. Thrombocytopenia was also seen in our patient [4]. In some cases with COVID-19 infection, the cough was not seen and symptoms of upper respiratory tract infection (rhinorrhea and sore throat) were rare [5]. In many studies, such as our study, levels of LDH, CRP, and AST increase and levels of white blood cells 
and lymphocytes decrease, which is indicative of COVID-19 [6]. It has been shown that LDH and CRP levels increase and decrease in children more than adults, respectively [7]. Most people with COVID-19 were male, which was different from our patient. It affects most middle-aged people. [8]. Oxygen therapy was used to improve respiratory status so that SPO2 was $90 \%$ to $95 \%$. People with COVID-19 positive for their nucleic acid test using RT-PCR and chest radiographs show patchy ground-glass opacity which was similar to our study. Crazy-paving pattern was found in some cases. Pure consolidation without GGO was less popular. In some patients, chest radiography was normal at the beginning of the disease [9]. In a study performed in China, $25 \%$ of people with COVID -19 had unilateral pneumonia and $75 \%$ of them had bilateral pneumonia. In our case, bilateral pneumonia were observed [5]. It has been shown that treatment with chloroquine and lopinavir/ritonavir (Kaletra) has a significant role in improving the condition of patients, which was consistent with our study [10]. Seeing this lesion on the leg, our patient initially suspected of having thromboembolism, which, after a CT scan, revealed a person with COVID-19, with no evidence of pulmonary thromboembolism. Deep Vein Thrombosis was suspected due to redness, pain, and tenderness at the leg veins, which was seen by color Doppler ultrasound in external iliac, common iliac, small saphenous, and large saphenous. None of the people with COVID-19 had this complication $[5,9,10]$. The blood clots of deep vein thrombosis can be caused by anything that prevents blood from circulating or clotting normally, such as injury to a vein, surgery, certain medications, and limited movement, but the cause of this complication is still unclear $[4,11]$. Heparin was used for the treatment of DVT and this procedure continued with rivaroxaban for prophylaxis [11]. In many studies, the diagnosis was made by X-rays, clinical signs, and blood tests, which, if any of the aforementioned methods did not contribute to the diagnosis of COVID-19, the disease was confirmed by the nucleic acid test [2]. RT-PCR should be carefully sampled. Types of samples include upper airway specimens (pharynx, nasal swabs, nasopharyngeal secretions), inferior airway specimens (sputum, air ducts, bronchoalveolar lavage fluid), blood, stool, urine, and conjunctival secretions. Sputum and other lower respiratory tract specimens are highly positive for nucleic acids and should preferably be collected from them. Combined detection of nucleic acids from several types of samples can improve the accuracy of detection. Among patients with confirmed positive nucleic acid in the respiratory tract, about $30 \%-40 \%$ of these patients have detected viral nucleic acid in the blood and about $50 \%-60 \%$ of the patients have viral nucleic acid in the stool. However, the rate of the positive nucleic acid test in urine samples is very low. Combined testing with respiratory, fecal, blood and other specimens is useful to improve the diagnostic sensitivity of suspected cases, monitor the effectiveness of treatment, and manage post-discharge isolation measures [10]. The mechanism of DVT formation due to COVID-19 is unknown despite thrombocytopenia, and has not been investigated but it resolved as COVID-19 symptoms, tenderness, and leg pain improved. Although COVID-19 presented with Deep Vein Thrombosis is a rare condition, in middle-aged people with sudden onset of manifestations, we should recognize it from other diseases as an important and treatable differential diagnosis. Rapid diagnostic assays, efficient treatment, and prudent use of CT-scan are important to control future COVID-19 spread.

\section{Abbreviations}


WHO: World Health Organization; COVID-19: Coronavirus disease 2019; SARS-Cov-2: Severe Acute Respiratory Syndrome Coronavirus 2; DVT: Deep Vein Thrombosis; AST: Aspartate Transaminase; LDH: Lactate Dehydrogenase; CRP: C- reactive protein; RT-PCR: Real Time - Polymerase Chain Reaction; SPO2: Spot Oxygen Saturation; ACA: Anti-cardiolipin antibodies; ANA: Antinuclear Antibody; Anti-dsDNA: Antidouble stranded DNA antibody; RF: Rheumatoid factor; GGO: ground-glass opacity.

\section{Declarations}

\section{Ethics approval and consent to participate}

Not applicable

\section{Consent for publication}

Informed written consent for publication was obtained from the patient prior to collecting information. The patient gave written consent for their personal or clinical details along with any identifying images to be published in this study.

\section{Availability of data and materials}

The datasets generated and/or analyzed during the current study may be made available from the corresponding author on a reasonable request.

\section{Competing interests}

The authors declare that they have no competing interests.

\section{Funding}

We did not receive any fund for this research

\section{Author's contributions}

AR analyzed the data and was a major contributor in writing the manuscript. MT performed the diagnostic tests. LD collected the clinical data. LD designed the case report and was responsible for communicating the work. All authors read and approved the final manuscript.

\section{Acknowledgments}

We would like to express our gratitude to the patient in the case and his daughter, who helped us in preparing the data.

\section{Author details}


${ }^{1}$ Assistant Professor of Infectious Diseases, Microbial Resistance Research Center, Mazandaran University of Medical Sciences, Sari

${ }^{2}$ Assistant Professor of Cardiology, Cardiovascular Research Center, Mazandaran University of Medical Sciences, Sari

${ }^{3}$ Medical Student, Student Research Committee, Faculty of Medicine, Mazandaran University of Medical Sciences, Sari, Iran.

\section{References}

1.Chen, H., et al., Clinical characteristics and intrauterine vertical transmission potential of COVID-19 infection in nine pregnant women: a retrospective review of medical records. The Lancet, 2020. 395(10226): p. 809-815.

2.Shen, K., et al., Diagnosis, treatment, and prevention of 2019 novel coronavirus infection in children: experts' consensus statement. World Journal of Pediatrics, 2020: p. 1-9.

3.Organization, W. H., Coronavirus disease 2019 (COVID-19): situation report, 51. 2020.

4.Lippi, G., M. Plebani, and B. M. Henry, Thrombocytopenia is associated with severe coronavirus disease 2019 (COVID-19) infections: A meta-analysis. Clinica Chimica Acta, 2020.

5.Huang, W.-H., et al., 2019 novel coronavirus disease (COVID-19) in Taiwan: Reports of two cases from Wuhan, China. Journal of Microbiology, Immunology and Infection, 2020.

6.Wang, D., et al., Clinical characteristics of 138 hospitalized patients with 2019 novel coronavirusinfected pneumonia in Wuhan, China. Jama, 2020.

7.Du, W., et al., Clinical Characteristics of COVID-19 in Children Compared with Adults Outside of Hubei Province in China. Available at SSRN 3546097, 2020.

8.Guan, W.-j., et al., Clinical characteristics of coronavirus disease 2019 in China. New England Journal of Medicine, 2020.

9.Cheng, S.-C., et al., First case of Coronavirus Disease 2019 (COVID-19) pneumonia in Taiwan. Journal of the Formosan Medical Association, 2020.

10.Chen, D., et al., Recurrence of positive SARS-CoV-2 RNA in COVID-19: A case report. International Journal of Infectious Diseases, 2020.

11.Tarannum, N., M. S. Azam, and R. K. Premchand, May-Thurner Syndrome and Recurrent DVT: A Case Report. Indian Journal of Clinical Cardiology, 2020. 1(1): p. 13-16. 


\section{Figures}

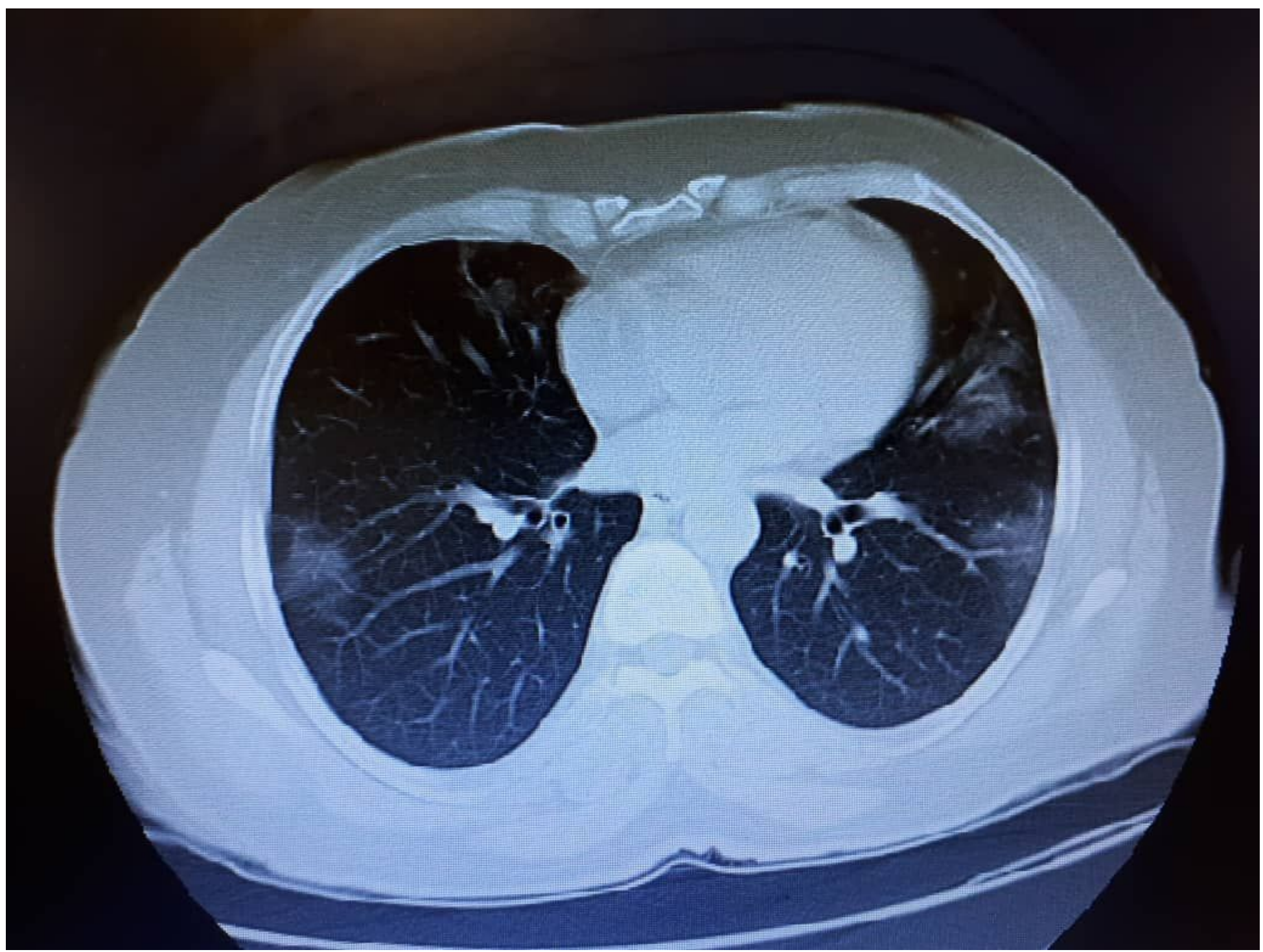

Figure 1

Chest CT showed persistent multifocal GGOs with or without superimposed reticulation 


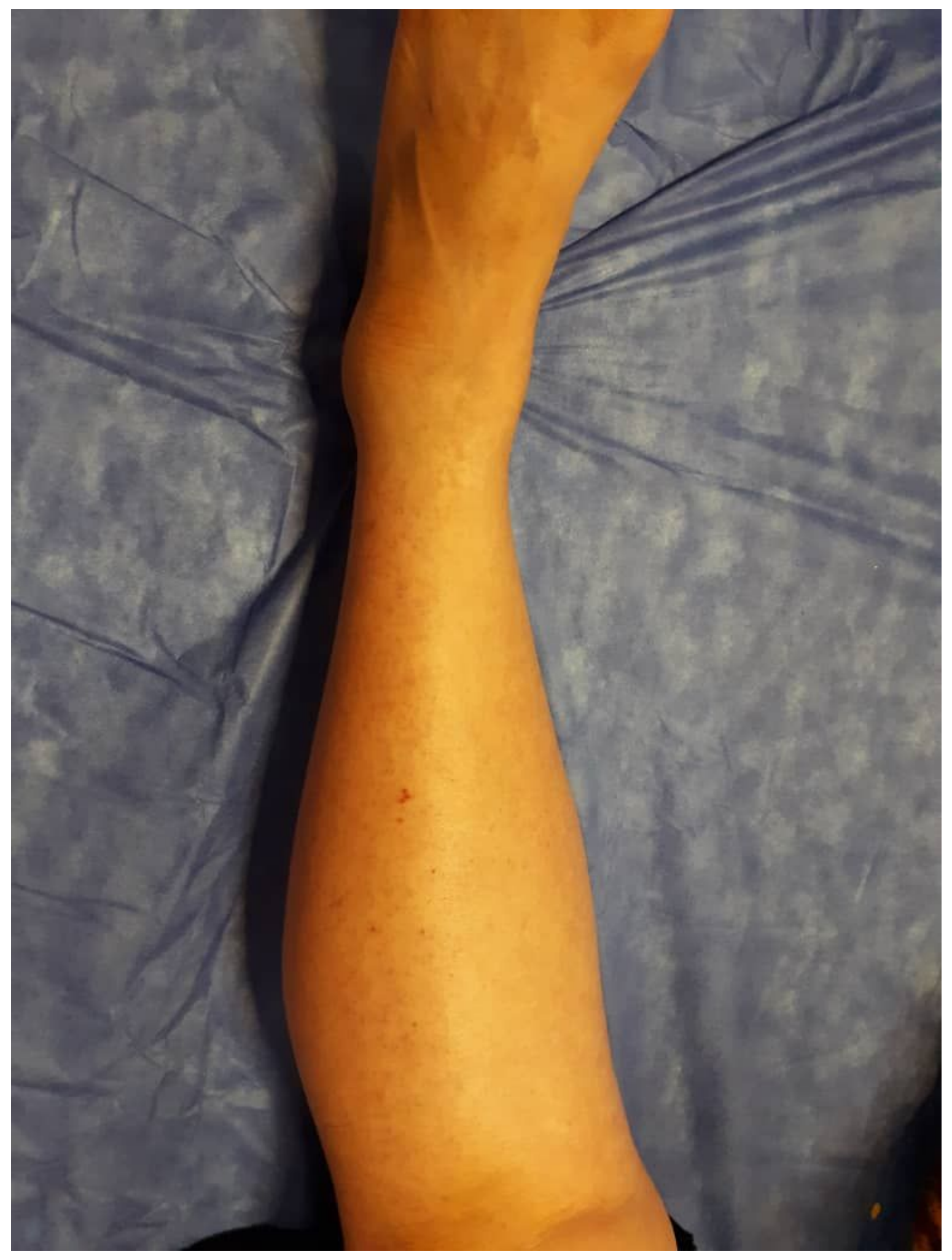

Figure 2

Clinical image of redness, and leg swelling due to DVT 


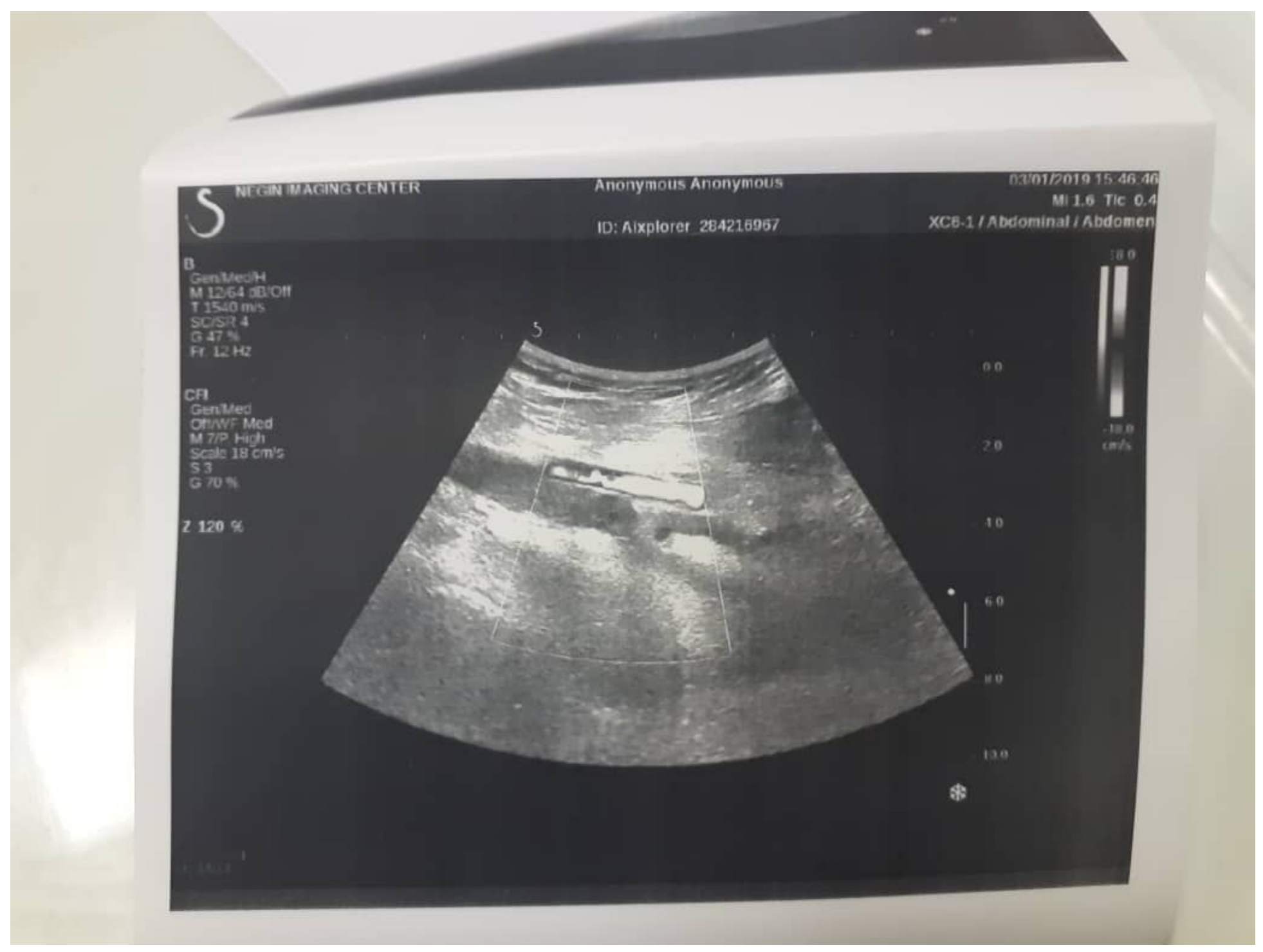

\section{Figure 3}

Abdominal color Doppler ultrasound image for DVT detection 